\title{
Viabilidad económica de proyectos REDD+ en la Amazonia brasileña
}

\section{Economic viability of REDD+ project in the Brasilian Amazonia}

\author{
Ana Milena Plata Fajardo', Romano Timofeiczyk Junior', Zenobio Abel Gouvêa Perelli da Gama e Silva², \\ João Carlos Garzel Leodoro da Silva', Anadalvo Juazeiro dos Santos' y Paula Andrea Mosquera Agudelo'
}

\footnotetext{
1 Universidade Federal do Paraná. Curitiba, Paraná, 2 Universidade Federal do Acre. Rio Branco, Acre, * Autor de correspondencia. aplatafa@gmail.com Brasil. Brasil.
}

\section{RESUMEN}

Este estudio tuvo como objetivo estimar la viabilidad y evaluar los riesgos económicos de los proyectos de Reducción de Emisiones por Deforestación y Degradación forestal - REDD+ en la Amazonia brasileña. Los datos fueron obtenidos a través de entrevistas y cuestionarios a los proponentes de proyectos privados de REDD+ en la Amazonia. El riesgo económico se calculó de acuerdo con un análisis de sensibilidad de las variables clave de los proyectos y la probabilidad de viabilidad de proyectos mediante el método de Simulación Monte Carlo. Los resultados obtenidos indican que los costes variables corresponden a $80 \%$, mientras que los fijos representaron $20 \%$ de los costos totales. Los resultados del análisis de riesgos sujetos a flujo de caja indican que los proyectos REDD+ en la Amazonia brasileña son viables, con Tasa Interna de Retorno (TIR) de 11,49\% por año, superior a la Tasa Mínima de Atractividad real de 10\% por año utilizada. El punto en el que el proyecto mostró un Valor Actual Neto (VAN) positivo es el precio de BRL 18,6; donde proyectos que vendan Verified Carbon Units (VCUs) a un precio inferior a BRL 18,6 podrán presentar inviabilidad financiera. La simulación de Monte Carlo mostró que la probabilidad de obtener una TIR de 10\% o más por año es 51,53\%. Se concluye que los proyectos de REDD+ en la Amazonia tienen una probabilidad de retorno positivo próximo a 50\%, pero esos retornos no llegan a ser tan atractivos en relación con el alto riesgo.

PALABRAS CLAVE: costos proyectos REDD+, evaluación de riesgo, flujo de caja, mercado de carbono, VCUs, simulación de Monte Carlo.

\section{ABSTRACT}

This study aims to estimate the profitability and to evaluate the economic risks of REDD + projects in the Brazilian Amazon. The data were obtained through interviews and questionnaires to the proponents of the private REDD + projects of Amazonia. The economic risk was calculated using sensitivity analysis of the key project variables and the probability of project profitability through the Monte Carlo Simulation Method. The results indicate that the variable cost was responsible for $80 \%$ of the total costs. Fixed costs accounted for $20 \%$ of total costs. The result of the cash flow risk analysis concluded that REDD + projects in the Brazilian Amazon are viable, Internal Rate of Return of $11.49 \%$ a.a. was greater than the $10 \%$ a.a. Minimum Attractiveness Rate. The point at which the project computes a positive NPV is the price of BRL 18.6. Projects that sold VCUs at a price lower than BRL 18.6 may be financially unfeasible. The Monte Carlo simulation showed that the probability of obtaining a TIR of $10 \%$ a.a. or more is $51.53 \%$ a.a. It is concluded that REDD + projects in the Amazon have a positive return probability approaching $50 \%$, but this return is not as attractive.

KEYWORDS: REDD+ cost, risk evaluation, cash flow, carbon market, VCUs, Monte Carlo simulation. 


\section{INTRODUCCIÓN}

Brasil está entre los cinco mayores emisores mundiales de gases de efecto invernadero, siendo que $61 \%$ de las emisiones son causadas por el cambio de uso del suelo y la deforestación. Para aumentar la inversión forestal, así como detener la deforestación, Brasil deberá identificar y evaluar las barreras que se dan en la inversión del sector ambiental (Ministerio de Ciencia y Tecnología [MCT], 2014). En este contexto, los proyectos de Reducción de Emisiones por Deforestación y Degradación forestal - REDD+ representan una alternativa económica para mitigar el cambio climático.

A pesar de estos esfuerzos, existe una limitación de los desarrolladores de proyectos REDD+ para expandir sus operaciones. Esto se debe a que proyectos REDD+ son, al principio, intensivos en capital y con largos ciclos de tiempo; además la falta de financiación también se produce en función de los riesgos de la naturaleza de estos proyectos. En consecuencia, los inversionistas, los bancos y los gobiernos están cada vez más preocupados por encontrar formas más precisas de evaluación de proyectos y programas. Otro elemento clave en las decisiones de inversión, que desempeña un papel decisivo en si un proyecto será o no capaz de atraer a los inversores, es el riesgo. Los proyectos REDD+ son comúnmente asociados con altos niveles de riesgo (Chenost et al, 2010). Por lo tanto, es necesario analizar más de cerca esta cuestión y aclarar cómo este riesgo afecta las decisiones de inversión del sector privado y cómo los proyectos pueden reducirlos para que se trasformen a ser financieramente atractivos.

En Brasil, se han hecho pocos progresos en relación con el análisis de los riesgos y beneficios en la implementación de proyectos REDD+, siendo escasos los estudios en el campo de evaluación, viabilidad, medición y análisis de riesgos. La literatura sobre el tema del calentamiento global revela que esa falta de estudios se debe a la escasez de datos y poca cooperación entre los ofertantes de proyectos REDD+.

Los altos costos para la ejecución de estos proyectos, el largo periodo para un posible retorno de capital y el riesgo no estimado dejan ver que la inversión es poco atractiva (Watson, 2013). En consecuencia, REDD + está subrepresentado como una clase de activos en grandes carteras de inversión (Glauner, Rinehart, D’Anieri, Boscolo y Savenije, 2012). En este sentido, son los estudios oportunos que proporcionan la información necesaria para facilitar una asignación más eficiente de los recursos para cumplir con los compromisos internacionales para reducir las emisiones de gases de efecto invernadero. Se tiene la necesidad de establecer criterios para medir el impacto económico de proyectos encaminados a reducir las emisiones a través del desarrollo de una herramienta para medir la inversión y el riesgo económico en el sector REDD+ en Brasil.

En la literatura, se pueden encontrar útiles estudios sobre los impactos ambientales en el riesgo de crédito (Graham y Maher, 2006; Schneider, 2011) y en las correlaciones entre los riesgos ambientales y la fijación de precios de servicios ecosistémicos (Drut 2010; Dwyer 2012; Scholtens 2010). Sin embargo, no hay un método científicamente fundamentado, que genere la información, permitiendo a los inversores identificar y gestionar los riesgos que puedan afectar a la inversión y el retorno de las inversiones en proyectos de REDD+ y cuantificar esos impactos en unidades monetarias con el fin de facilitar la integración.

A pesar de las limitaciones e incertidumbres sobre las variables que influyen en la rentabilidad de los proyectos REDD+, este estudio trata de llenar el vacío, buscando identificar y evaluar el riesgo económico de los proyectos REDD + en Brasil.

\section{OBJETIVOS}

El objetivo general de este trabajo es estimar la viabilidad y evaluar el riesgo económico de los proyectos de REDD+ en la Amazonia brasileña. Para ello, se plantean los siguientes objetivos específicos: 1) cuantificar y analizar la estructura de costos del proyecto REDD+; 2) evaluar los riesgos económicos asociados a la variabilidad de los componentes de flujo de efectivo de los proyectos REDD+; 3) determinar el precio, la cantidad de VCUs y el 
área mínima para permitir un proyecto REDD+; y 4) determinar el precio, la cantidad de VCUs y el área mínima para viabilizar un proyecto REDD+.

\section{MATERIALES Y MÉTODOS}

En este estudio se consideraron siete proyectos REDD+ situados en la Amazonia, validados y verificados en los estándares Verified Carbon Standard [VCS] y Climate, Community and Biodiversity Alilance [CCBA] ${ }^{1}$. En la tabla 1 se resumen la ubicación, el año, las VCUs anuales, las áreas y, en general, las principales características de los proyectos contemplados en este trabajo.

De acuerdo con la tabla 1, se puede observar que el proyecto REDD+ que ha generado más VCUs es Envira, el cual se encuentra en Acre, en el municipio de Feijó y fue desarrollado por Carbon Fund. Por otro lado, el proyecto Envira no es el más grande en área. El proyecto más grande en términos de área es el proyecto Florestal Santa María que fue desarrollado en un área de 72338 hectáreas, en el municipio Colniza en Mato Groso (Verified Carbon Standard [VCS], 2015).

Se utilizó como referencia el área promedio de los siete proyectos analizados y el promedio de las VCUs generadas por hectárea como un aproximado para el diseño del proyecto estándar REDD+ en la Amazonia. De acuerdo con Toginho y Andrello (2009), los proyectos estándar son proyectos con características similares que tienen como objetivo generar un modelo y homogeneizar especificaciones, costos y formas de ejecución.

De acuerdo con el promedio de los proyectos investigados, el proyecto base que se analizó tuvo un área de 41700 hectáreas y genera 9,4 VCUs por hectárea.

\section{Recolección de datos}

La fuente de datos de esta investigación se divide en fuente de datos primarios y fuente de datos secundarios.

1 Los estándares VCS y CCB son los más utilizados para proyectos REDD + en Brasil y en los países tropicales (The REDD DESK, 2016).
Fuente de datos primarios. Contiene los datos de todos los recursos físicos y financieros necesarios para la implantación de proyectos REDD+. A continuación, se presentan las fuentes de datos primarios:

1. Entrevistas estructuradas. Se realizaron entrevistas a empresas Biofilica, Carbon Fund, Sustainable Carbon, Florestal Santa Maria S.A., Oreades, que son los licitadores de los proyectos y fueron considerados en este estudio

2. Cuestionario. El cuestionario tuvo como objetivo recoger datos sobre la estructura de costos, las perspectivas del mercado, las barreras institucionales y financieras de los proyectos. El proyecto fue enviado a los siete proyectos REDD+ de la Amazonia.

Fuente de datos secundarios. Los datos secundarios de este estudio fueron obtenidos de los siete VCS y de investigaciones cuantitativas planeadas por Ecosystem Market Place (2016). A continuación, se presentan las principales fuentes de datos secundarios:

1. Documento de concepción de proyecto (DCP). Para que un proyecto sea registrado en el mercado de carbono debe ser presentado de acuerdo con una metodología previamente establecida por el Panel Intergubernamental sobre Cambio Climático [IPCC]. El DCP representa la fuente más unificada de información, ya que contempla características requeridas por metodologías existentes. La documentación del proyecto plausible consiste en completar el expediente necesario con el fin de hacer que los proyectos sean atractivos para los inversionistas. El DCP se coloca en el sitio web de estándares validadores de proyectos, con el fin de proporcionar un proceso transparente en los criterios de los DCP. 
2. Investigaciones cuantitativas de Ecosystem Market Place. Ecosystem Market Place publica información anual sobre las transacciones cuantitativas del mercado de carbono forestal, así como otros mercados de servicios ambientales. La investigación de Ecosystem Market Place es extremadamente importante en el estudio, ya que es la fuente más confiable sobre el precio de la transacción de VCUs.

El procedimiento metodológico correspondió a análisis de viabilidad y de riesgo; para este propósito se definió la estructura de costos y la formación de flujo de caja. Las evaluaciones del proyecto estiman los costos y beneficios, a menudo con bajo riesgo debido a las limitaciones de los datos en el modelo de flujo de caja. La identificación y caracterización adecuada de este riesgo es una parte integral de la segunda etapa de esta investigación metodológica. Las metodologías utilizadas para evaluar el riesgo fueron: análisis de sensibilidad, mejor y peor escenario, el punto de equilibrio y la simulación Monte Carlo.

Se utilizó el análisis de riesgos para evaluar el efecto de la variación de ciertos inputs en los resultados y la viabilidad de los proyectos. Mediante el modelo de Monte Carlo, se tuvo la intención de obtener un contexto más preciso de los resultados, destacando las limitaciones del análisis actual y auxiliar a los tomadores de decisiones en la comprensión de los impactos potenciales de las opciones de beneficios y costos.

\section{Precio}

Los precios de VCUs son el resultado de una negociación bilateral entre el ofertante y el demandante, es decir, los proponentes de los proyectos y los compradores de VCUs. Los proponentes de proyectos analizados no mencionaron el precio de venta de sus VCUs en el cuestionario, ya que esto puede perjudicar negociaciones y ventas futuras.

Para el presente estudio se consideraron los precios publicados por la investigación de los Ecosystem Market Place (2016) que organiza una serie histórica de un año, donde se verifica una alta volatilidad en los precios de los VCUs. El precio para los proyectos de REDD+ en el año 2016 fue, en promedio, de RBL 19,47 por VCU. Este valor de RBL 19,47 por VCU fue obtenido del informe Ecosystem Marketplace (2016).

TABLA 1. Localización y características cuantitativas de los proyectos REDD+ en la Amazonia.

\begin{tabular}{|c|c|c|c|c|c|c|c|c|}
\hline $\begin{array}{l}\text { Nombre del } \\
\text { proyeto }\end{array}$ & Año & Estado & Auditor & Estándar & $\begin{array}{l}\text { Área } \\
\text { (ha) }\end{array}$ & $\begin{array}{l}\text { VCU * } \\
\text { anual }\end{array}$ & $\begin{array}{l}\text { VCUs * } \\
\text { porha }\end{array}$ & Proponente \\
\hline $\begin{array}{l}\text { Florestal } \\
\text { Santa Maria }\end{array}$ & 2012 & $\begin{array}{l}\text { Mato } \\
\text { Grosso }\end{array}$ & $\begin{array}{l}\text { Imaflora/ } \\
\text { Rainforest Alliance }\end{array}$ & VCS & 72000 & 997444 & 13,85 & $\begin{array}{l}\text { Plant } \\
\text { environment } \\
\text { intelligent }\end{array}$ \\
\hline $\begin{array}{l}\text { Jari/Amapá } \\
\text { REDD+ }\end{array}$ & 2013 & Pará & Scs global services & VCS & 65980 & 115009 & 1,74 & Biofilica \\
\hline Rusas & 2014 & Acre & $\begin{array}{l}\text { Environmental } \\
\text { services,inc }\end{array}$ & $\mathrm{VCS}+\mathrm{CCB}$ & 42000 & 120147 & 2,86 & Carbon CO \\
\hline $\begin{array}{l}\text { Envira } \\
\text { Amazonia }\end{array}$ & 2014 & Acre & $\begin{array}{l}\text { Environmental } \\
\text { services,inc }\end{array}$ & $\mathrm{VCS}+\mathrm{CCB}$ & 39300 & 1259646 & 32,05 & Carbon CO \\
\hline Purus & 2012 & Acre & $\begin{array}{l}\text { SCS Global } \\
\text { Services }\end{array}$ & $\mathrm{VCS}+\mathrm{CCB}$ & 35000 & 89868 & 2,56 & Carbon $\mathrm{CO}$ \\
\hline Valparaiso & 2014 & Acre & $\begin{array}{l}\text { Environmental } \\
\text { services,inc }\end{array}$ & $\mathrm{VCS}+\mathrm{CCB}$ & 28096 & 153583 & 5,47 & Carbon CO \\
\hline Ecomapua & 2012 & Pará & TUV Rheinland & VCS & 9600 & 72338 & 7,53 & $\begin{array}{l}\text { Sustainable } \\
\text { carbon }\end{array}$ \\
\hline
\end{tabular}

* VCU $=$ Verified Carbon Units

Fuente: elaboración propia, 2016. 
El informe recoge datos a través de una encuesta anual, monitoreando las transacciones en el momento exacto en que fueron realizadas. La información se basa en datos recogidos por los proponentes del proyecto, mayoristas, minoristas y corredores, así como compensación de carbono en los registros contables de las empresas.

\section{Ingresos}

El ingreso bruto corresponde al producto de la cantidad de VCUs generada anualmente y el precio negociado. Los VCUs se generan después de la validación de la información DCP de la norma de auditoría. La validación de la información es la aceptación de la metodología utilizada para estimar la reducción de las emisiones netas durante los 30 años. El proyecto REDD+ se aprobó sobre las metodologías estándar VCS, no es suficiente solo estimar el carbono reservado, el proyecto debe demostrar adicionalmente el cálculo de las fugas y demostrar la permanencia, además de otros criterios técnicos de acuerdo con la metodología y puede ser investigado con profundidad en el VCS (2015).

\section{Evaluación de riesgo}

La primera fase del proceso de análisis de riesgo es la determinación de un modelo robusto capaz de predecir los resultados. El análisis de costo-beneficio en este estudio incluyó una evaluación de riesgos, para determinar el nivel de este en la variable. El análisis de viabilidad contempló escenarios asociados con las variables no determinadas.

Antes de describir los métodos utilizados de análisis de riesgos, es importante reconocer que para este estudio se identificaron variables deterministas y estocásticas. Las variables de riesgo son fundamentales para el análisis del proyecto, donde una pequeña variación puede alterar significativamente el resultado. Después de este análisis, se utilizaron las técnicas de evaluación de riesgo, tales como análisis de sensibilidad, los mejores y los peores escenarios, el análisis del punto de equilibrio y análisis de Monte Carlo.

\section{Escenarios optimistas y pesimistas}

$\mathrm{El}$ análisis de escenarios tuvo en cuenta los valores mínimos de todas las variables y describe lo que fue la variación del VAN y del TIR. Por lo que el análisis de escenarios considera los valores máximos de las variables y mostró cómo se alteró el VAN y el TIR sobre este supuesto (Institut for Miljovurdering / Environmental Assessment Institute [IMV], 2006). Antes de realizar el análisis de escenarios es necesario estimar los valores mínimos, medios y máximos de los proyectos REDD+ en Brasil. Para este fin, se utilizó la información de los siete proyectos REDD+ en Brasil que se incluyeron en la encuesta.

\section{Simulación Monte Carlo}

Una simulación de Monte Carlo se utilizó para analizar el impacto de algunos de los componentes de flujo de caja del VAN. El producto bruto de análisis de riesgos Monte Carlo fue una serie de resultados organizados y presentados en forma de distribución de probabilidad de los posibles resultados del proyecto. Esto por sí solo es una imagen muy útil del perfil de riesgo/rendimiento del proyecto que puede mejorar la decisión de inversión. Solo mediante la comprensión del proceso del proyecto, se puede mejorar la posibilidad de la venta de créditos de carbono y finalmente garantizar la sostenibilidad financiera de los proyectos.

Para este estudio, se determinó el riesgo antes de que el modelo se elaborara y reflejara las características de las variables aleatorias. La primera tarea correspondió a la estimación de valores futuros de las variables proyectadas. En la práctica, la definición de los valores de las variables proyectadas se puede hacer mediante la estimación de distribución de probabilidad. Las distribuciones de probabilidad fueron definidas teniendo en cuenta el perfil de probabilidad de ocurrencia en proyectos anteriores.

A menudo, la evaluación de la inversión se calculan tres valores en los resultados del flujo de caja, un valor promedio, un escenario pesimista y un optimista (Savvakis, 1994). Del mismo modo, se incorporaron las correlaciones entre los componentes, se incorporaron y determinaron matemáticamente sus características aleatorias. 
La definición de los valores medios, máximos y mínimos se realizó de acuerdo con la información recogida en los cuestionarios y en observaciones históricas de las variables. Esta información se utilizó para determinar la distribución y frecuencia de ocurrencia. La distribución de probabilidad, en este caso, es la distribución de la frecuencia expresada en términos relativos que van de 0 a 1 .

Después de la determinación de las probabilidades, se simuló por un gran número de rondas a través de Excel, lo cual mostró distribuciones de probabilidad (histogramas) de VAN y la TIR. Durante la simulación, los valores de las variables de riesgo se seleccionaron al azar dentro del modelo de costo-beneficio de acuerdo con distribuciones de probabilidad y, en el proceso de simulación, se construyeron escenarios sucesivos. Los resultados del modelo fueron computarizados y se almacenaron después de cada simulación y los histogramas de VAN representan la probabilidad de que el proyecto tiene un VAN mayor que cero (o cualquier otro valor).

Para determinar el intervalo de las variables, fueron consultados los proponentes que sugerían intervalos de valores probables de las variables estocásticas. Cuando la variable fue la suma de las contribuciones de varios expertos, los argumentos teóricos para la elección se definieron sobre la distribución normal. Cuando los valores máximo y mínimo, así como un valor "central" o promedio estuvieran disponibles de la distribución triangular, se determinó que sería una opción conveniente. Las variables que tienen valores discretos fueron consideradas por distribuciones discretas. Finalmente fueron consideraras las variables que entraron en el cálculo del VAN y están correlacionadas.

$\mathrm{El}$ análisis de Monte Carlo permitió evaluar el riesgo de efectos sobre las variables clave, teniendo en cuenta las posibles correlaciones entre ellos. Se trata de sustituir entradas individuales con distribuciones de probabilidad de valores posibles.

\section{Crystal Ball}

Crystal Ball es una herramienta complemento de Microsoft Excel que ayuda a la simulación de Monte Carlo. El software Crystal Ball se utilizó para simular el VAN y la TIR de los proyectos REDD+ y ayudó a determinar la base lineal del modelo y las variables que influyen en la viabilidad de los proyectos. Crystal Ball mejora el flujo de dinero en efectivo, permitiendo crear distribuciones de probabilidad que describen la incertidumbre en torno a las variables de entrada específicas. Para este estudio se incluyeron cuatro distribuciones de probabilidad, haciendo referencia en Crystal Ball como "supuestos". Fue elegida la distribución triangular por ser el que mejor refleja las características de las variables.

En el número de repeticiones para las simulaciones se determinaron 1000 simulaciones, como fue recomendado por el IMV (2006). Cada simulación diseña una distribución de probabilidad de todas las variables y calcula el VAN. El ajuste de la distribución simulada para la distribución teórica mejora a medida que el número de simulaciones aumenta.

\section{RESULTADOS Y DISCUSIÓN}

El proyecto de norma REDD+ en el Amazonas tuvo ingresos de RBL 7880256 anuales. Es decir, ingresos de RBL 189 por hectárea por año. Es importante tener en cuenta que casi la totalidad de la inversión se produce en el primer año, la cual es de RBL 5823065 lo que sumado a las inversiones del año cero (RBL 1064 111) representa una inversión total de RBL 6887 176. Los criterios de evaluación del proyecto (VAN y TIR) utilizados en el análisis económico mostraron que el proyecto REDD+ en el Amazonas es económicamente viable. La TIR del proyecto REDD+ en la Amazonia mostró un valor más alto $(11,49 \%$ por año) que el de TMA (10\% por año), lo que hace viable el proyecto y el VAN de este es 831 605, mayor que cero.

Otros proyectos que se pueden desarrollar en la Amazonia brasileña consisten en la siembra de las especies frutales, la gestión de sistemas agroforestales y la venta de Cotas de Reserva Ambiental (CRA). En cuanto al análisis de viabilidad de otros emprendimientos, Lima Junior, Rezende y Oliveira (1997) estiman una TIR de $24 \%$ por año 
y un ingreso de RBL 2050 por año por hectárea para la siembra de açaí en la Amazonia.

Del mismo modo, los resultados de Barbosa et al. (2015), al analizar el sistema integrado agropecuario y forestal en Brasil, se encontró que la TIR de estos sistemas se encuentra entre $13,99 \%$ por año y $14,95 \%$. Por su parte, el Instituto Internacional De Sustentabilidade [IIS] (2015) sobre la viabilidad del sector pecuario en la Amazonia, encontró resultados positivos para la producción ganadera, con una TIR de 15,13\% por año (teniendo en cuenta el $10^{\circ}$ año de producción). Sin embargo, el IIS (2015) encontró que los ingresos de los emprendimientos de ganado vacuno son de USD 2638 comparado con una granja de 1000 ha, lo que supone unos ingresos de RBL 263. Con respecto a las CRA, el rendimiento por hectárea está valorado en USD 3094 por año (Guerreiro, 2016). Estos resultados de otros proyectos en la Amazonia brasileña son mucho mayores que las que se encuentran para proyectos de REDD+. Sin embargo, la comparación es limitada debido a las diferentes condiciones de los proyectos, como las tasas críticas de rentabilidad, el horizonte de planificación, el riesgo, entre otros. Una comparación real no es por tanto, posible, debido a estas condiciones que varían.

\section{Escenarios optimistas, realistas y pesimistas}

El resultado ilustra los límites superior e inferior del resultado del análisis de costo-beneficio. El siguiente ejemplo ilustra cómo afecta la viabilidad del proyecto en el supuesto más negativo y el flujo de caja más positivo.

Escenario optimista. Un escenario optimista en el Amazonas es generar 13 VCUs por hectárea, es decir, de acuerdo con el proyecto de Envira en Acre, considerando una superficie de 41700 hectáreas, esto significaría una generación de 542100 VCUs por proyecto. La estimación se realizó considerando que si aumentó la cantidad de VCUs también eleva el costo de registro, y la tasa de trasferencia aumenta, de esta forma los costos de operación referentes a las actividades para reducir la deforestación, ya que, si el proyecto genera más VCUs, significa que la línea base del proyecto fue menor; es decir, la presión de la deforestación en la zona era mayor y por lo tanto también aumentará el costo de la reducción de la deforestación. Las otras variables que no estén correlacionadas con la cantidad de VCUs se consideraron condiciones ceteris paribus. Con estos escenarios positivos de una mayor cantidad de VCUs registrada en un proyecto REDD+ en la Amazonia, el proyecto logra una TIR de $26 \%$ por año. Es importante aclarar que este escenario es muy poco probable, solamente 14\% de los proyectos REDD+ en la Amazonia generan esa cantidad de VCUs.

Escenario realista. El escenario más probable corresponde a los valores medios de generación VCUs, es decir, 9,62 por hectárea. Teniendo en cuenta la superficie media de 41711 hectáreas, esto significaría una generación de 401186 VCUs por proyecto. El flujo de caja de un proyecto REDD+ en la Amazonia, considerando el escenario más probable, significa la generación media de VCUs por hectárea, los costos operativos promedio, las medianas de los gastos operativos, los costos de transacción promedio y el precio medio de venta de VCUs, que se estima una TIR de 11,49\% por año.

Escenario pesimista. El escenario más negativo corresponde a la generación de 2,57 VCUs por hectárea. Considerando una superficie media de 41700 hectáreas, esto significaba una generación de 107169 VCUs por proyecto. En este escenario, el VAN muestra resultados negativos en la TIR.

Las entrevistas con los proponentes del proyecto revelaron que la mayoría de los inversores privados en Brasil financian proyectos REDD+ para fines de Responsabilidades Sociales Corporativas (RSC), sin expectativas reales de rendimiento. Muchas otras empresas han financiado proyectos REDD+ con la esperanza de reducir su huella de carbono y por lo tanto evitar la penalización de los gobiernos. Sin embargo, no todas las empresas están sujetas a las regulaciones de reducción de emisiones, porque no todos los países tienen un mercado de carbono o las mismas normas de regulación de la materia. Crear políticas para el desarrollo de los mercados de carbono y alentar al sector privado a participar 
considerablemente, reduce el riesgo de los proyectos REDD+.

Para una empresa, los proyectos REDD+ no son la única manera de convertirse en "carbono neutral". El aumento de la eficiencia energética, la promoción de tecnologías limpias y materiales de reciclaje son algunos ejemplos de reducción de las emisiones corporativas, lo que puede no ser atractivo para todas las empresas que invierten en proyectos REDD+. La inversión en proyectos de REDD + es atractiva cuando el precio para reducir una tonelada de $\mathrm{CO}_{2}$ es menor que la reducción de la misma tonelada de $\mathrm{CO}_{2}$ a través de la adopción de tecnologías limpias.

\section{Punto de equilibrio}

Para generar 223985 VCUs, las empresas de REDD+ necesitan tener un ingreso de BRL 4360997 por año, para por lo menos no tener pérdidas financieras y por encima de este valor ya se tienen ganancias. En cuanto al precio, el punto en el que el proyecto calcula un VAN positivo y una
TIR mayor al TMA (10\% por año) es el precio de RBL 18,6. Proyectos que deciden vender VCUs a un precio inferior a USD 18,6 puedan presentar inviabilidad financiera. Este modelo se ejemplifica en la figura 1 , desde un punto de vista teórico.

\section{Simulación Monte Carlo}

La simulación Monte Carlo mostró que la probabilidad de lograr un TIR mayor que $10 \%$ por año es de $51,53 \%$ (Fig. 2). Los análisis de histograma de las mil simulaciones mostraron una frecuencia del TIR de los porcentajes acumulados; los resultados muestran una TIR positiva y la distribución se mostró simétrica. Esto es muy importante, ya que cuando los estudios empíricos de la serie de datos financieros tienen asimetría y/o exceso de curtosis, el riesgo está subestimado (Vasques, 2005).

En relación con este estudio, la aproximación a la distribución normal significa que la media es igual a la moda y la mediana y es posible inferir un intervalo de confianza de 95\% en los resultados (Bittencourt y Vialli, 2006).

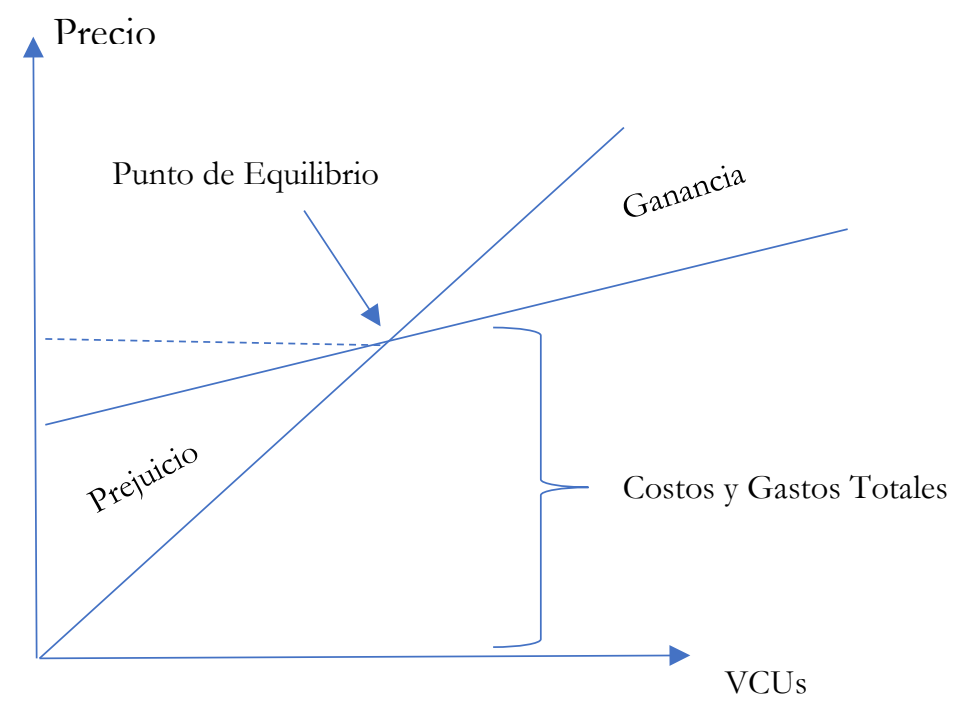

FIGURA 1. Modelo de análisis del punto de equilibrio financiero para los proyectos. 


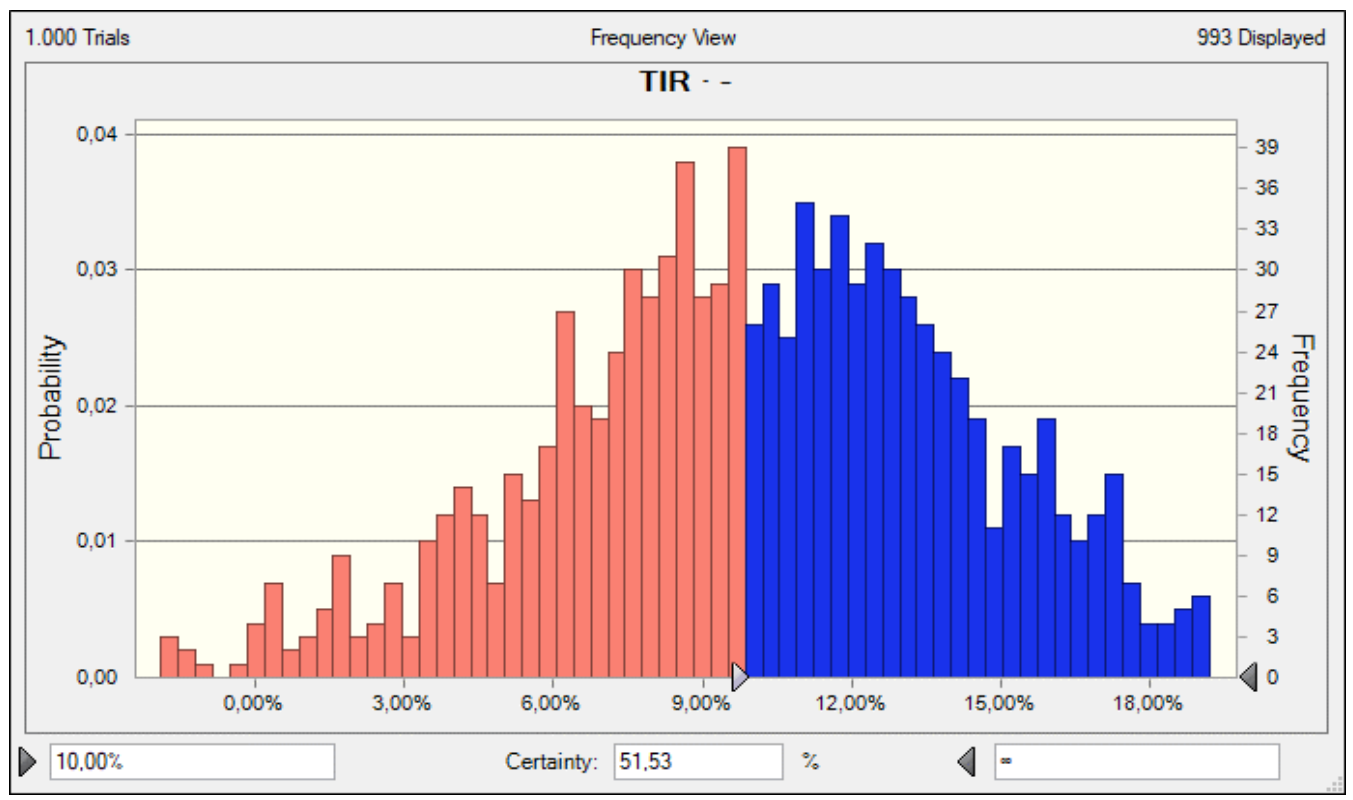

FIGURA 2. Simulación Monte Carlo TIR Fuente: elaboración propia, 2017.

Para Coelho Júnior, Rezende, Oliveira, Coimbra, Souza (2008), basados en el método de Monte Carlo, las decisiones mostraron más de $50 \%$ de probabilidad de ocurrencia, las decisiones tomadas son ya más altas que las que se toman en los métodos tradicionales de las evaluaciones económicas. Por lo tanto, cuanto más conocimiento y dominio de los escenarios del mercado tiene el gestor del proyecto, más precisa será su toma de decisiones (Araujo, Santos, Nogueira, Lima y Silva 2013). Debido al hecho de que el proyecto REDD+ tiene estrechas probabilidades de viabilidad, hasta de $50 \%$, se recomienda prudencia en la inversión en los proyectos de la Amazonia.

En relación con el riesgo de otros proyectos en la Amazonia, se presenta el sector pecuario. El estudio de IIS (2015) llevó a cabo un análisis de Monte Carlo para sistemas de ganado en la Amazonia, bajo la variación del precio de venta de carne y los costos de producción (costos fijos, costos variables). También se rodaron 1000 interacciones y los resultados concluyeron que la probabilidad de obtener una viabilidad en una finca de 1000 es de $76,33 \%$, mientras que la probabilidad de obtener una viabilidad en una finca de 4000 es de 85,25\%; es decir, que la probabilidad de un aumento en un VAN positivo aumenta a medida que crece el tamaño del proyecto en el sector pecuario.

El sector pecuario, en relación con los de proyectos REDD+, tiene algunas ventajas porque hay menos vulnerabilidad a las variaciones climáticas estacionales y hay poco control de los costos de depreciación (Barbosa et al., 2015). Como se mencionó en el estudio de viabilidad, el sector pecuario arroja una TIR entre $13,99 \%$ por año y $14,95 \%$ por año, pero a pesar de que este sector en la Amazonia muestre valores similares a la TIR, la cual se pueda comparar con proyectos REDD+ (11,49\% por año), las probabilidades de rentabilidad en el sector pecuario son mucho mayores que en los proyectos REDD+, 85,3\% en comparación con 51,27\%. Sin embargo, el desarrollo de estos proyectos pecuarios en la Amazonia ha sido impulsado desde los años sesenta, cuando el gobierno brasileño construyó la vía Belem-Brasilia, la actual BR-364 y Transamazónica, además de establecer el Instituto Nacional da Colonização e Reforma Agrária [INCRA], sin embargo, el primer proyecto privado de REDD+ en la Amazonia fue en el año 2012. 
La escasez de mejores prácticas en el campo y la solidez de análisis en el desempeño productivo y financiero son las razones que dificultan la inversión por parte de los empresarios rurales en los proyectos de REDD+. El proceso de desarrollo económico de la Amazonia, en los últimos años, ha promovido la deforestación en la región y el sector pecuario ha sido identificado como el principal agente responsable de esto para reemplazar las áreas forestales por cultivos de pastos.

Sería interesante analizar cómo estos valores están por encima o por debajo de otros resultados de los proyectos REDD+, pero hasta la fecha no se encuentra otro estudio de simulación Monte Carlo para este tipo de proyectos. El criterio de decisión para llevar a cabo un proyecto sobre estos valores de riesgo también depende de factores psicológicos de los inversores. Un riesgo del inversor adverso no aceptará un retorno positivo con una probabilidad cercana a $40 \%$, ya que prefiere asignar sus recursos en sector pecuario o en una cuenta bancaria con una tasa interna de rentabilidad más baja, pero garantizada, en vez de optar por acciones que pueden tener un alto rendimiento, pero también una probabilidad de perder su valor.

Inversores con tolerancia de riesgo pueden invertir en proyectos con probabilidades cercanas a $50 \%$ y que resulte en altos rendimientos positivos. Sin embargo, este no es el caso del proyecto de REDD+ en la Amazonia, ya que presenta probabilidades positivas de retorno cerca de $50 \%$, pero el rendimiento no es tan atractivo. Con una TIR baja y un riesgo cercano a 50\% del retorno positivo, el inversor más tolerante optó por no invertir.

\section{CONCLUSIONES}

- La estructura de los proyectos REDD+ en la Amazonia revela que el costo variable es mayor que el costo fijo en relación con los salarios y cargos.

- A diferencia de los proyectos forestales no madereros, los costos variables de los proyectos REDD+ representan el mayor costo unitario debido a la exigencia de mano de obra especializada. La preparación del documento de diseño de proyecto contempla el uso de metodologías aceptadas y reconocidas por la norma internacional. Los costos de mano de obra podrían reducirse si en el futuro hay normas nacionales para proyectos REDD+ en Brasil.

- Los impuestos representan un alto porcentaje de la proporción de costo variable total (25,4\%). La alta carga tributaria en Brasil no estimula proyectos REDD $+y$ actualmente no hay incentivos fiscales para los nuevos proyectos.

- De acuerdo con la simulación de Monte Carlo la probabilidad de obtener Tasa Interna de Rendimiento mayor que la Tasa Mínima de Atracción (10\% por año) es $51,53 \%$. Estos valores no son atractivos para los inversores.

- El proyecto es de alto riesgo, por lo que los inversores de riesgo intolerantes no pueden invertir porque el rendimiento no es alto en comparación con otros proyectos forestales.

- El mercado voluntario de carbono se está posicionando en América Latina por la demanda de las empresas que necesitan para compensar el uso de combustibles fósiles y así evitar el pago de impuestos relacionados con esta actividad. El impuesto carbono en Colombia, Chile y México es de USD 5 (RBL 16,5); sin embargo, de acuerdo con el análisis de viabilidad REDD + los proyectos del Amazonas son viables en el supuesto de precios promedio de VCUs (RBL 19.47). Esto significa que las VCUs de Brasil no son económicamente atractivas en los mercados latinoamericanos.

- El proyecto es de alto riesgo, por lo que el inversor de riesgos intolerantes no puede invertir porque el rendimiento no es alto en comparación con otros proyectos forestales.

- También se deduce que las proyecciones de la tasa de cambio del dólar para el año 2018 es alta y puede causar un aumento en los ingresos de VCUs, lo que podría hacer viable el proyecto. Por otro lado, los costos de mano de obra y la preparación de los proyectos REDD+ pueden caer por la capacidad técnica del brasileño para este tipo de proyectos, y los precios de las VCUs pueden incrementar mediante el aumento de la 
demanda de proyectos REDD+ en el mundo, a través de normas más estrictas sobre la reducción de emisiones. Esta última perspectiva positiva permite a los proponentes de proyectos REDD+ estar atentos a las condiciones del mercado para ejecutar proyectos viables.

- El punto de equilibrio financiero reveló que el precio de venta de VCU mínimo para que el proyecto sea viable es todavía alto. Hecho que confirma por qué las nuevas empresas no han entrado en el mercado voluntario de carbono forestal en Brasil en los últimos dos años

- Brasil tiene una ventaja sobre el área mínima para proyectos REDD+ en la Amazonia. El punto de equilibrio de la zona (38 $717 \mathrm{ha}$ ) no es un factor limitante para la estructuración de proyectos. Es más fácil encontrar áreas de más de 15 módulos fiscales en la Amazonia, con aptitud forestal para proyectos REDD+ que en otros países tropicales como Colombia.

- Los proyectos de REDD+ se caracterizan por altos niveles de riesgo, lo que los hace poco atractivos para los inversores privados, especialmente los inversores adversos al riesgo.

El mercado de carbono, en particular para los proyectos REDD+, es relativamente nuevo y solo comenzó a desarrollarse a partir del año 2012. Los proyectos privados se han desarrollado en la Amazonia, esto significa que el mercado todavía no ha alcanzado la madurez y su desarrollo sigue estando en las primeras etapas.

\section{REFERENCIAS}

Araujo, L., Santos, M., Nogueira, A., Lima, K. \& Silva, A. (2013) Método Monte Carlo Aplicado em Florestas Energéticas. Enciclopédia Biosfera. Centro Científico Conbecer - Goiânia, 9(17), 1883-1897.

Barbosa, F., Monteiro, P., Andrade, V., Batista, G., Maciel, I. \& Queiroga, S. (2015). Avaliação econômica e produtiva dos sistemas integrados de lavoura-pecuária-floresta. Caderno de Cadencies Agrarias, 7(1), 151-165.

Bittencourt, H. \& Viali, L. (2006) Contribuições para o Ensino da Distribuição Normal ou Curva de Gauss em Cursos de Graduação. III Seminário Internacional de Pesquisa em Educação Matemática.
Coelho Júnior, L. M., Rezende, J. L. P. de, Oliveira, A. D. de, Coimbra, L. A. B. \& Souza, A. N. (2008). Análise de investimento de um sistema agroflorestal sob condição de risco. Cerne, Lavras, 14(4) 368-378.

Chenost, C., Gardette, Y., Demenois, J., Grondard, N., Perrier, M. \& Waemere, M. (2010) Les marchés du carbone forestier. UNEP, ONFI, AFD, BioCF.

Drut, B. (2010) Sovereign Bonds and Socially Responsible Investment. Journal of Business Ethics, 92(1), 131-145.

Dwyer, A. (2012) Sustainability and sovereign credit ratings in emerging markets: Nigeria as a case study. Recuperado de http://dx.doi.org/10.2139/ssrn.2147533

Ecosystem Market Place (2016). Raising Ambition State of the Voluntary Carbon Markets.

Glauner, R., Rinehart, J. A., D’Anieri, P., Boscolo, M., \& Savenije, H. (2012). Timberland in institutional investment portfolios: Can significant investment reach emerging markets? Working Paper No. 31. Roma: FAO.

Graham, G. \& Maher, J. (2006). Environmental liabilities, bond ratings, and bond yields. Volume Advances in Environmental Accounting \& Management, 3, 111-142.

Guerreiro, I. (2016). Cota de Reserva Ambiental pode gerar mais de R\$5 bilhões na Amazônia. Recuperado de http:/ / amazonia.org.br/2016/05/cota-de-reserva-ambientalpode-gerar-mais-de-r-5-bilhoes-na-amazonia/

Institut for Miljøvurdering / Environmental Assessment Institute [IMV] (2006). Risk and uncertainty in cost benefit analysis. Toolbox paper.

Instituto Internacional De Sustentabilidade [IIS] (2015). Análise econômica de uma pecuária mais sustentável. Recuperado de http://www.iisrio.org/publicacoes

Lima Junior, V., Rezende, J. \& Oliveira, A. D. de (1997) Determinação da taxa de desconto a ser usada na análise econômica de projetos florestais. Cerne, Lavras, 3(1), 45-66.

Ministério De Ciência E Tecnologia [MCT] (2014). Estimativas anuais de emissões de gases de efeito estufa no Brasil.

Savvakis S. (1994) Risk analysis in investment appraisal. Project Appraisal, 9(1), 3-18. doi: 10.1080/02688867.1994.9726923

Schneider, T. E. (2011) Is environmental performance a determinant of Bond Pricing? Evidence from the U.S. pulp and paper and chemical industries, Contemporary Accounting Research, 28(5), 1537 1561. doi: 10.1111/j.1911-3846.2010.01064.x

Scholtens, B. (2010) The environmental performance of Dutch Government Bond Funds. Journal of Business Ethics, 92(1), 117130. doi:10.1007/s10551-010-0637-4 
The REDD DESK. Verified Carbon Standard | Jurisdictional and Nested REDD+ (VCS JNR) Recuperado de: http:// theredddesk.org/markets-standards/verified-carbonstandard-jurisdictional-and-nested-redd-vcs-jnr

Toginho, D. E., \& Andrello, A., (2009) Catálogo de Experimentos do Laboratório Integrado de Física Geral Departamento de Física. Universidade Estadual de Londrina.

Vasques, B. (2005) Modelos baseados em Simulação de Monte Carlo: Soluções para o cálculo do Value-at-Risk. Dissertação de Mestrado. Universidade Federal do Rio de Janeiro Instituto COPPEAD de Administração, Brasil.

Verified Carbon Standard [VCS]. (2015) AFOLU Projects. Recuperado de http://www.v-c-s.org/develop-project/agriculture-forestryprojects

Watson, A. (2013) How to measure the performance of forestry projects? Recuperado de https://openforests.com/building-bridgesimpact-investors-sustainable-forestry
Manuscrito recibido el 28 de agosto de 2018

Aceptado el 20 de junio de 2019

Publicado el 11 de abril de 2020

Este documento se debe citar como:

Fajardo, A. M. P., Timofeiczyk Junior, R., Silva, Z. A. G. P. G., Garzel L. S., J. C., Juazeiro S., A., \& Mosquera A., P. A. (2020). Viabilidad económica de proyectos REDD+ en la Amazonia brasileña. Madera y Bosques, 26(1), e2611761. doi: 10.21829/myb.2020.2611761

Madera y Bosques por Instituto de Ecología, A.C. se distribuye bajo una Licencia Creative Commons Atribución-NoComercialCompartirlgual 4.0 Internacional. 